\section{Detection of mitochondrial DNA mutations in patients with diabetes mellitus}

\section{Dear Sir,}

The role of mitochondrial DNA (mtDNA) mutations in diabetes continues to excite interest. Blanché et al. [1] have recently suggested that the use of a non-isotopic method (PhastSystem, Pharmacia, Uppsala, Sweden), offers significant advantages over previously reported methods of mutation detection. However, their communication raises a number of important issues.

Firstly, pathogenic mtDNA mutations are often readily detected by polymerase chain reaction (PCR) amplification and specific restriction digestion. The resultant products are run on an ethidium-stained agarose gel. This does not involve radio-isotopic PCR and is quicker, cheaper and more robust than the use of the PhastSystem. The problem of false-negatives will only arise in patients with very small $(<10 \%)$ proportions of mutated mtDNA in peripheral leucocytes. Studies of patients with mitochondrial myopathies suggests that the likelihood of missing mutations using non-isotopic PCR is small [2].

The PhastSystem employs silver-staining to detect DNA and this is known to be a sensitive method [3]. However, it is equally possible to use silver staining on standard non-PhastSystem gels. Blanché et al. do not comment on whether by using the PhastSystem they were able to detect the mutation in any subject where the defect could not have been detected by simple ethidium-stained gels. Neither do they make a comparison between the pre-cast PhastSystem gels and non-PhastSystem silver stained gels.

In our opinion, the main advantage of the PhastSystem is in its use for heteroduplex and single stranded conformational polymorphism (SSCP) analysis. The nature of the pre-cast gels and the precise running conditions allow rapid and accurate detection of point mutations. We have recently developed the use of the PhastSystem for the detection of mutations in mtDNA [4].

Froguel et al. $[5,6]$ have previously published articles on the use of the PhastSystem for the detection of nuclear mutations and the use of isotopic PCR for the mitochondrial 3243 bp mutation. Despite the title of their current communication, they provide no objective evidence for the sensitivity of the technique. We have sought to rectify this omission.

Mitochondrial DNA was isolated from a subject known to have a heteroplasmic mutation. The $\mathrm{tRNA}^{\mathrm{leu}(\mathrm{UU} \mathrm{R})}$ region was

Corresponding author: Dr. J. C. Alcolado, Department of Medicine, University Hospital of Wales, Cardiff CF4 4XW, UK amplified by PCR as previously described [7]. We then diluted the $\mathrm{PCR}$ product known to harbour the mutation with various volumes of wild-type PCR product (from a normal control without the mutation), to produce ratios of mutant: wild-type PCR product ranging from $50 \%$ mutant:50\% wild-type to $100 \%$ wild-type. The mixed PCR products were denatured and run on the PhastSystem using conditions designed to detect the SSCP produced by the mutation [4]. Pre-cast gels were silver stained using the Pharmacia kit. As shown in Figure 1 , we were able to detect the SSCP produced by the mutation at a ratio of less than $1 \%$. The true sensitivity will be greater than this, since the sample with the mutation will not have had $100 \%$ mutant DNA in peripheral leucocytes.

This result, together with those of our other work [4] confirm that the PhastSystem is a rapid and reliable system for the detection of mtDNA mutations causing SSCPs. For the simple screening of large numbers of subjects for known diabetes-associated mutations (e.g. the 3243 bp mutation) we advocate simple non-isotopic PCR followed by the ethidium-

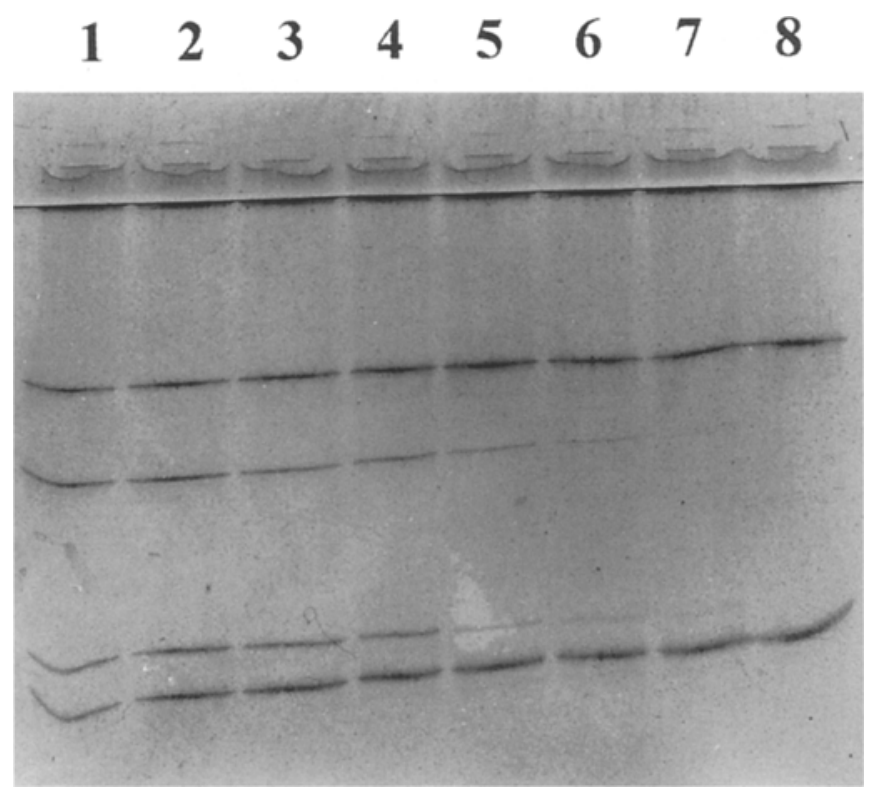

Fig.1. The sensitivity of mitochondrial heteroplasmic mutation detection by SSCP analysis using the Pharmacia PhastSystem and silver staining. PCR products from wild type (wt) and a known mutant $(\mathrm{mt})$ were mixed to give final wt:mt percentage ratios of; (1) $50: 50$, (2) $60: 40$, (3) $80: 20$, (4) $90: 10$, (5) $95: 5$, (6) $98: 2$, (7) $99: 1$ and (8) 100:0. Samples were electrophoresed on a $20 \%$ gel at $300 \mathrm{~V}, 2.5 \mathrm{~mA}, 20 \mathrm{~W}, 11^{\circ} \mathrm{C}$ for $1000 \mathrm{Vh}$ 
staining of agarose gels. In cases where clinical suspicion is high, for example in patients with a strong maternal history of diabetes, isotopic-PCR or silver staining of either standard gels or PhastSystem gels may be appropriate, but only if nonisotopic methods are negative.

Yours sincerely,

A. W. Thomas, R. Morgan, A. Majid, A. Rees, J. C. Alcolado

\section{References}

1. Blanché H, Froguel Ph, Dausset J, Cohen D, Cohen N (1994) Non-isotopic and sensitive method for diagnosis of maternally-inherited diabetes and deafness. Diabetologia 37: 842

2. Hammans SR, Sweeney MG, Brockington M et al. (1994) Mitochondrial encephalomyopathies; molecular genetic diagnosis from blood samples. Lancet 337: 1311-1313

\section{Selection of primers for detection of $A$ to $G$ mutation at nucleotide 3243 of the mitochondrial gene}

Dear Sir,

Blanché and colleagues [1] report a sensitive method for the diagnosis of maternally inherited diabetes with an A-to-G mutation at nucleotide 3243 of the mitochondrial gene. We also used almost the same method to detect this mutation (unpublished data). However, we think another selection of the primers for polymerase chain reaction (PCR) amplification may lead to an increased possibility of detecting the mutation. We compared the detection rate of this mutation of two sets of primers for amplification: (1H) AGGACAAGAGAAATAAGGCC (3130-3149), (1L) CACGTTGGGGCCTTTGCGTA (3423-3404), and (2H) CGTTTGTTCAACGATTAAAG (3035-3054), (2L) AGCGAAGGGTTGTAGTAGCC (3456-3437). Using the second set of primers the two cleaved fragments (212bp and 214 bp) moved to almost the same position in the gel, resulting in a more distinct single band. Because the population of the mu-

Corresponding author: Dr. M.Odawara, Institute of Clinical Medicine, The University of Tsukuba, Tsukuba, Japan
3. Beidler JL, Hilliard PR, Rill RL (1992) Ultrasensitive staining of nucleic acids with silver. Anal Biochem 126: 374-380

4. Thomas AW, Morgan R, Sweeney M et al. (1994) The detection of mitochondrial DNA mutations using single stranded conformational polymorphism (SSCP) analysis and heteroduplex analysis. Human Genetics 94: 621-624

5. Hager J, Blanche H, Sun et al. (1994) Six mutations in the glucokinase gene identified in MODY by using a non-radioactive sensitive screening technique. Diabetes 43: 730-733

6. Vaxillaire M, Vionnet V, Vigouroux C et al. (1994) Search for third susceptibility gene for maturity onset diabetes of the young; studies with eleven candidate genes. Diabetes 43: 389-395

7. Alcolado JC, Majid A, Brockington et al. (1994) Mitochondrial gene defects in patients with NIDDM. Diabetologia 37: $372-376$

tant DNA of the mitochondrial genome in the peripheral blood is relatively low, thin bands may be overlooked in the screening for this mutation. Although the use of labelled primers is also useful for the detection of this mutation, the procedures are more complicated. From our experience we recommend using the second set of the primers. At the first screening of 300 Japanese patients with non-insulin-dependent diabetes using the $(1 \mathrm{H})$ and $(1 \mathrm{~L})$ primers, we only found two patients positive for the mutation. However, the second set of primers detected one more patient with this mutation.

Retrospectively, the mutation was found to be overlooked because of the thinness of the two bands. We also recommend using polyacrylamide gel or PhastSystem (Pharmacia, Uppsala, Sweden), for the analysis rather than agarose gel, because although expensive it can obtain more distinct bands.

Yours sincerely,

M. Odawara, K.Sasaki, Y.Tachi, K. Yamashita

\section{References}

1. Blanché H, Froguel Ph, Dausset J, Cohen D, Cohen N (1994) Non-isotopic and sensitive method for diagnosis of maternally-inherited diabetes and deafness. Diabetologia 37: 842 (Letter) 